\title{
EFFECT OF MAGNETIC FIELD ON FINE STRUCTURE OF TUNNEL CURRENT IN DOUBLE-BARRIER RESONANT-TUNNELING DEVICES
}

\author{
A.E. Belyaev, S.A. Vitusevich, B.A. Glavin \\ Institute of Semiconductor Physics, National Academy of Sciences of Ukraine \\ Kiev 252028, Ukraine
}

\author{
A. MąKosa AND W. Dobrowolski \\ Institute of Physics, Polish Academy of Sciences \\ Al. Lotników 32/46, 02-668 Warszawa, Poland
}

An effect of magnetic field on a fine oscillatory structure revealed in the resonant current flowing through double-barrier resonant-tunneling devices is examined. It is found that the observed variation of the fine structure in a magnetic field parallel to the current direction differs considerably from that appearing in tunnel current flowing through single-barrier structures. Experimental results are explained in terms of the quantum interference effect arising in structures having wide spacer layers.

PACS numbers: 73.40.Gk, 85.30.Mn

Recently [1], we reported the observation of a fine oscillatory structure in the tunnel current flowing through double-barrier resonant-tunneling structures (DBRTS). This feature was treated in terms of the sequential LO-phonon emission by electrons transmitted through DBRTS. Our further studies of DBRTS incorporating various kinds of spacer layers in both emitter and collector parts revealed a number of peculiarities in the oscillatory picture. Firstly, similar oscillations for both polarities of the bias voltage have been observed. The oscillations are clearly seen up to $120 \mathrm{~K}$ and their amplitude increases monotonously with applied voltage. In some structures the oscillation period is considerably smaller than $36 \mathrm{mV}$ which corresponds to LO-phonon energy. Moreover, changes in the fine structure observed in DBRTS, caused by applied magnetic field, differ considerably from the ones discovered in single barrier structures [2] where oscillations in tunnel current were explained mostly by the sequential LO-phonon emission although other explanations have also been proposed. Similar explanation seems 
to be not appropriate to our case, first of all, because the observed period of oscillations (which, moreover, refers to the total voltage drop across the structure) is too small to take account for the LO-phonon scattering process.

To explain the above-mentioned peculiarities, we suggest a model based on the quantum interference effect that can arise in structures having a wide spacer layer. The model predicts the appearance of a fine structure in the tunnel current whose period of oscillations is determined solely by the size parameters of the structure. The discussed interference effect differs from that which occurs in wide quantum wells and which leads to the appearance of a great number of resonance peaks in the current-voltage characteristic (CVC) [3]. In our case the quantum interference of electrons is assumed to take place in accumulation and depletion layers on both sides of the barriers. Their length in our structures is of the order of $100 \mathrm{~nm}$, so the transport in them is ballistic. These regions act as resonators for electron wave and impose their fine structure on the resonant transparency of the double-barrier system. An applied voltage shifts a resonant level in the well with respect to this fine structure which gives rise to weak oscillations in CVC. It should be noticed that in some systems with specific doping profile quantum properties of electron transport lead to a stronger effect, namely to bistability of CVC even at zero bias [4].

Ilere, the period of oscillations is determined by the potential distribution which, in turn, is established by the doping profile. For its calculation it is necessary to solve the Schrödinger equation self-consistently with the Poisson equation. It is a rather complicated task but some qualitative results may be obtained in a simplified model. We assume further the accumulation layer to form a triangular well. When a spacer layer exists in the emitter region, its length determines the size of the triangular well. The well has to be deep enough to store a sufficient number of electrons that is necessary to build up an appropriate electric field in the barriers. If there is no emitter spacer, it is impossible to determine parameters of the well as simply as in the previous case. In that case the current oscillations can arise from electron interference in the wide spacer layer on the collector side of the structure as shown by calculations.

The Schrödinger equation has been solved for the case of triangular well and the net current was obtained after averaging over the Fermi distribution. The result of such calculations is presented in Fig. 1. We have really found oscillations although the number of cycles is considerably smaller than that observed in the experimental characteristics (compare the curve at $0 \mathrm{~T}$ in Fig. 2). Most probably this disagreement is due to oversimplification in our model; after all it is not self-consistent. Nevertheless, the model permits to explain the appearance of oscillations in tunnel current having the period smaller than that corresponding to the LO-phonon energy. To check the validity of the model we carried out experiments in a magnetic field parallel to the current flow. Experiments were done with DBRTS incorporating various kinds of spacer layers on both emitter and collector sides. All the structures display a qualitatively very similar behaviour. For that reason we will henceforth discuss one of them. This structure consists of the following layers: substrate, $n^{+}-\mathrm{GaAs}(100)$-oriented; buffer, GaAs doped with $\mathrm{Si}$, $n=10^{18} \mathrm{~cm}^{-3}, 100 \mathrm{~nm}$; spacer, GaAs undoped, $100 \mathrm{~nm}$; barrier, AlAs undoped, 

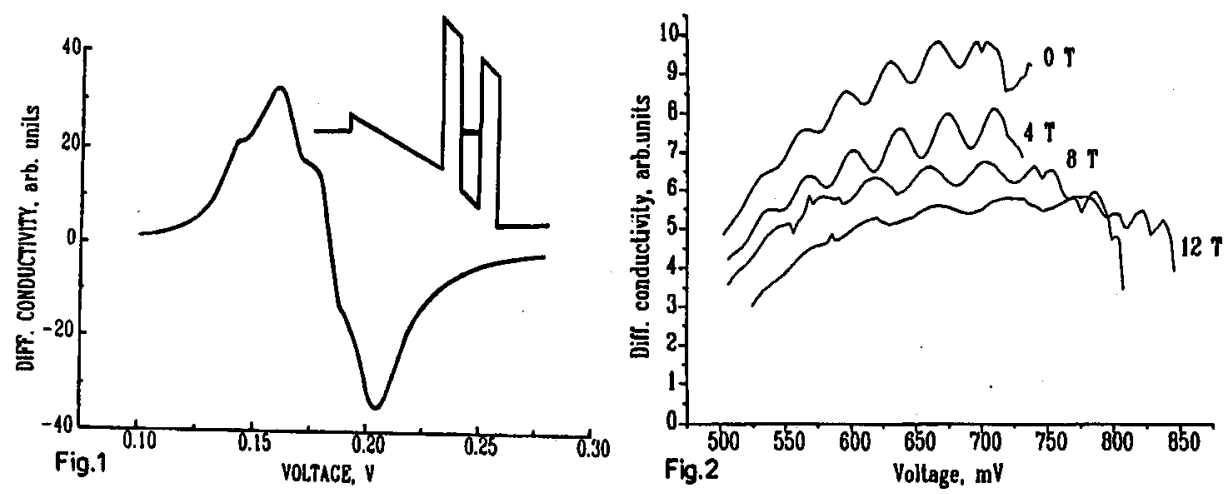

Fig. 1. Calculated dependence of differential conductance on applied voltage. The inset shows the model potential profile for the structure with a wide emitter spacer layer.

Fig. 2. Dependence of differential conductance on applied voltage in the range of resonance peak for different magnetic field inductions.

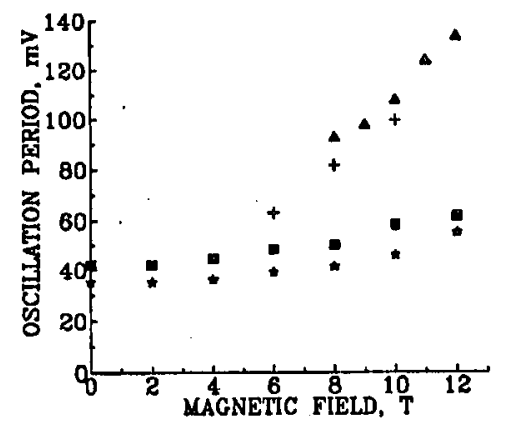

Fig. 3. Magnetic field dependence of the period of oscillations appeared on the increasing slope of the first (stars) and the second (squares) of the two resonance peaks observed in the investigated DBRTS under the forward bias. Triangles correspond to magneto-oscillations observed in the same structure in the valley-current region. Crosses represent data from Ref. [5].

$2 \mathrm{~nm}$; well, GaAs undoped, $4 \mathrm{~nm}$; barrier, AlAs undoped, $2 \mathrm{~nm}$; top contact, GaAs doped with $\mathrm{Si}, n=10^{18} \mathrm{~cm}^{-3}, 100 \mathrm{~nm}$.

Figure 2 shows the differential conductance, $d I / d V$, of the structure in the region of resonance peak for different magnetic fields when the substrate is biased negatively (forward bias). In a magnetic field exceeding $6 \mathrm{~T}$ the fine structure weakens and shifts to a higher bias voltage. Simultaneously the period of oscillations increases slightly which is clearly seen in Fig. 3 (curves marked with stars and squares). At the same time the period of magneto-oscillations that arise in the valley-current region increases almost linearly with the magnetic field (curve marked with triangles in Fig. 3). These oscillations consist of several cycles whose maxima positions converges, as the magnetic field goes to zero, to a voltage corresponding to the LO-phonon replica of the resonance peak. Oscillations on the 
increasing slope of the resonant-tunneling current reported in Ref. [5] (marked with crosses in Fig. 3) display a similar behaviour. These both kinds of oscillations correspond to electron tunneling via Landau levels in the well and their period variation reflects a change in the cyclotron energy with the magnetic field. Thus, the much weaker variation of the period of the fine oscillatory structure, observed by us on the increasing slope of the resonant-tunneling current, should be explained by a mechanism which is not connected with the Landau quantization. In the frames of the quantum interference model, magnetic field affects the period of oscillations indirectly through the magnetic-field-induced changes in the potential profile of the structure. The only fact remains not fully understood, namely, why the amplitude of oscillations decreases with the magnetic field and this problem is the subject of further investigations.

This work was supported by the State Committee of Science and Technology of Ukraine, grant No 7.01.05/141 and partly by the State Committee for Scientific Research (Republic of Poland), grant No 2 P03B 03508.

\section{References}

[1] S.A. Vitusevich, T. Figielski, A. Mąkosa, T. Wosiński, A.E. Belyaev, R.V. Konakova, L.N: Kravchenko, Acta Phys. Pol. A 87, 377 (1995).

[2] T.W. Hickmott, P.M. Solomon, F.F. Fang, F. Stern, Phys. Rev. Lett. 52, 2053 (1984).

[3] E.S. Alves, M.L. Leadbeater, L. Eaves, M. Henini, O.H. Hughes, Solid State Electron. 32, 1627 (1989).

[4] K.K. Gullapalli, A.J. Tsao, D.P. Neikirk, Appl. Phys. Lett. 62, 2856 (1993).

[5] A. Nogaret, L.A. Cury, D.K. Maude, J.C. Portal, D.L. Silco, A.Y. Cho, G. Hill, Semicond. Sci. Technol. 8, 1810 (1993). 